\title{
QUADRATIC INTEGRATION OF GAUSSIAN PROCESSES
}

\author{
T. F. LIN
}

\begin{abstract}
Let $x(t), 0<t<T$, be a Gaussian process whose covariance function $R(s, t)$ satisfies certain conditions. If $G(x)$ satisfies some mild condition, then the quadratic integral $L^{2}$-lim $\Sigma_{k} G\left(x\left(t_{k}\right)\right) \Delta x\left(t_{k}\right)^{2}$ along any sequence of paritions of $[0, T]$ whose mesh goes to zero exists. The differential rule for $x(t)$ is also derived.
\end{abstract}

0. Introduction. Let $x(t), 0 \leqslant t \leqslant 1$, be a Gaussian process with covariance function $R(s, t), 0 \leqslant s, t \leqslant 1$. In studying some connections between Gaussian processes and boundary value problems, Baxter showed, under certain conditions, (see [1, Theorem 1, p. 522], or [6, Theorem 20.4, p. 297]) that the quadratic variation of $x(t)$ over $[0,1]$ is

$$
\lim _{n \rightarrow \infty} \sum_{k=0}^{2^{n}-1} \Delta x\left(t_{k}\right)^{2}=\int_{0}^{1} f(t) d t
$$

where $t_{k}=t_{k}^{n}=k 2^{-n}, 0 \leqslant k \leqslant 2^{n}, \Delta x\left(t_{k}\right)=x\left(t_{k+1}\right)-x\left(t_{k}\right), 0<k<2^{n}-1$, and where $f(t), 0<t<1$, is defined by

$$
f(t)=\lim _{h \uparrow 0}\{R(t+h, t)-R(t, t)\} / h-\lim _{h \downarrow 0}\{R(t+h, t)-R(t, t)\} / h .
$$

This result was later extended to a wider class of processes (see [5], [2], [4]). The limit in (0.1) is $L^{2}$-convergent (as well as almost sure convergent). If $G(y)$ is a nice function of $y$, it is not hard to prove that

$$
\lim _{n \rightarrow \infty} E\left\{\sum_{k=0}^{2^{n}-1} G\left(x\left(t_{k}\right)\right) \Delta x\left(t_{k}\right)^{2}\right\}=E\left\{\int_{0}^{1} G(x(t)) f(t) d t\right\} .
$$

From (0.1) and (0.3), it is reasonable to conjecture that the quadratic integral of $G(x(t))$ w.r.t. $x(t)$ is

$$
L^{2}-\lim _{n \rightarrow \infty} \sum_{k=0}^{2^{n}-1} G\left(x\left(t_{k}\right)\right) \Delta x\left(t_{k}\right)^{2}=\int_{0}^{1} G(x(t)) f(t) d t .
$$

We shall prove that this is really the case under some suitable conditions.

Some basic properties of normal distributions are listed in $\S 1$. The proof of $(0.4)$ is given in $\$ 2$. As an application, Ito's lemma for $x(t)$ is derived in the last section.

1. Basic properties. In this section, we list some properties of Gaussian distribution which will be needed in the next section. Since the proofs of these properties are routine, they are omitted.

Received by the editors September 14, 1979 and, in revised form, March 25, 1980.

AMS (MOS) subject classifications (1970). Primary 60G15, 60G05.

Key words and phrases. Gaussian process, covariance function, quadratic integral, Ito's formula. 
Property 1.1. Let $X$ be a normal random variable with mean 0 and variance $\sigma^{2}$. Then for each $m>0$, there exists a constant $c_{m}$ such that $E\left\{\left(X^{2}-\sigma^{2}\right)^{m}\right\}=c_{m} \sigma^{m}$.

Property 1.2. Let $Y_{1}, Y_{2}$ have a bivariate normal distribution with means $\mu_{1}, \mu_{2}$ and variances $\sigma_{1}^{2}, \sigma_{2}^{2}$ respectively. Let $\rho$ denote their covariance. Then

$$
E Y_{1}^{2} Y_{2}^{2}=\sigma_{1}^{2} \sigma_{2}^{2}+2 \rho^{2}+\mu_{1}^{2} \sigma_{2}^{2}+\mu_{2}^{2} \sigma_{1}^{2}+4 \mu_{1} \mu_{2} \rho+\mu_{1}^{2} \mu_{2}^{2}
$$

Let random variables $Z_{i}, 1 \leqslant i<4$, have a multivariate normal distribution with zero means. Let $B=\left(b_{i j}\right)$ denote their covariance matrix and let $B_{i j}$ denote the cofactor of $b_{i j}$ for each $i, j$. Let $C=\left(c_{i j}\right)$ denote the covariance matrix of $Z_{1}, Z_{2}, Z_{3}$ and let $C_{i j}$ denote the cofactor of $c_{i j}$. Then we have

Property 1.3. The conditional density $N\left(z_{1}, z_{2} \mid z_{3}, z_{4}\right)$ of $Z_{1}, Z_{2}$ given $Z_{3}=z_{3}$, $Z_{4}=z_{4}$ is bivariate normal. Let $m_{1}, m_{2} ; V_{1}, V_{2} ; \Gamma$ denote the conditional means, variances and covariance respectively. Then

$$
\begin{gathered}
m_{1}=\left\{\left(B_{12} B_{23}-B_{13} B_{22}\right) z_{3}+\left(B_{12} B_{24}-B_{14} B_{22}\right) z_{4}\right\} /\left(B_{11} B_{22}-B_{12}^{2}\right), \\
m_{2}=\left\{\left(B_{12} B_{13}-B_{11} B_{23}\right) z_{3}+\left(B_{12} B_{14}-B_{11} B_{24}\right) z_{4}\right\} /\left(B_{11} B_{22}-B_{12}^{2}\right), \\
V_{1}=B_{22}|B| /\left(B_{11} B_{22}-B_{12}^{2}\right), \quad V_{2}=B_{11}|B| /\left(B_{11} B_{22}-B_{12}^{2}\right) \\
\Gamma=B_{12}|B| /\left(B_{12}^{2}-B_{11} B_{22}\right)
\end{gathered}
$$

where $|B|$ denotes the determinant of $B$.

ProperTy 1.4. The conditional density $N\left(z_{1}, z_{2} \mid z_{3}\right)$ of $Z_{1}, Z_{2}$ given $Z_{3}=z_{3}$ is bivariate normal. Let $m_{1}^{*}, m_{2}^{*} ; V_{1}^{*}, V_{2}^{*} ; \Gamma^{*}$ denote the conditional means, variances and covariance respectively. Then

$$
\begin{gathered}
m_{1}^{*}=\left(C_{12} C_{23}-C_{13} C_{22}\right) z_{3} /\left(C_{11} C_{22}-C_{12}^{2}\right), \\
m_{2}^{*}=\left(C_{12} C_{13}-C_{11} C_{23}\right) z_{3} /\left(C_{11} C_{22}-C_{12}^{2}\right), \\
V_{1}^{*}=C_{22}|C| /\left(C_{11} C_{22}-C_{12}^{2}\right), \quad V_{2}^{*}=C_{11}|C| /\left(C_{11} C_{22}-C_{12}^{2}\right), \\
\Gamma^{*}=C_{12}|C| /\left(C_{12}^{2}-C_{11} C_{22}\right),
\end{gathered}
$$

where $|C|$ denotes the determinant of $C$.

2. Main result. Throughout the rest of this article, let $x(t), 0<t<T$, denote a Gaussian process with mean function $m(t)$ and covariance function $R(s, t)$. Assume that $m(t)$ has a bounded first derivative for $0<t<T$ and that $R(s, t)$ satisfies conditions

(R1) $R(s, t)$ is continuous in $(s, t)$ and has continuous first partial derivatives in $\{(s, t): 0 \leqslant s \leqslant t \leqslant T\}$ (including one-sided partial derivatives at the boundary).

$(\mathrm{R} 2)|R(s+d s, t+d t)-R(s+d s, t)-R(s, t+d t)+R(s, t)| \leqslant$ constant $|d s d t|$ for $s \neq t$.

(R3) $R(t, t) R(s, s)-R(s, t)^{2}>0$ for $s \neq t$ (this condition will be dropped later, see Remark 1).

Condition (R1) implies that $f(t)$, as defined by (0.2), exists and is bounded and continuous for $0<t<T$. One can check easily that $f(t)$ is nonnegative. 
THEOREM 2.1. Let $G$ be a polynomial or a bounded continuous function. Then the $L^{2}$-limit of $\Sigma_{k} G\left(x\left(t_{k}\right)\right) \Delta x\left(t_{k}\right)^{2}$, along any sequence of partitions $P_{n}=\left\{t_{k}\right\}, n>1$, of $[0, T]$ whose mesh $d_{n}$ goes to 0 , exists and equals $\int_{0}^{T} G(x(t)) f(t) d t$. (Recall $\Delta x\left(t_{k}\right)=$ $x\left(t_{k+1}\right)-x\left(t_{k}\right)$.)

We shall prove Theorem 2.1 for the case $m(t) \equiv 0$ only. For the case $m(t) \neq 0$, the proof is similar to that of the special case $G(x) \equiv 1$ (see [1, pp. 524-525]). For each partition $P_{n}=\left\{t_{k}\right\}$, let $a_{i j}=E\left\{\Delta x\left(t_{i}\right) \Delta x\left(t_{j}\right)\right\}$. Then conditions (R1) and (R2) imply

$$
\left\{\begin{array}{l}
a_{i j}=O\left(\Delta t_{i} \Delta t_{j}\right), \quad i \neq j, \\
a_{k k}=f\left(t_{k}\right) \Delta t_{k}+o\left(\Delta t_{k}\right),
\end{array}\right.
$$

where the Landau notations $O(\cdot)$ and $o(\cdot)$ are uniform in $i, j, k$ as $d_{n} \rightarrow 0$ and where $\Delta t_{m}=t_{m+1}-t_{m}$ for $m=i, j, k$.

Given $\varepsilon>0$, condition (R3) implies that there exists $M_{\varepsilon}>0$ such that, for $|t-s| \geqslant \varepsilon$

$$
R(t, t) R(s, s)-R(s, t)^{2}>M_{\varepsilon} \text {. }
$$

Let $I_{\varepsilon}=\left\{(i, j):\left|t_{i}-t_{j}\right|>\varepsilon, f\left(t_{i}\right)>\varepsilon, f\left(t_{j}\right)>\varepsilon\right\}$. For $(i, j) \in I_{\varepsilon}$, let $z_{1}=\Delta x\left(t_{i}\right)$, $z_{2}=\Delta x\left(t_{j}\right), z_{3}=x\left(t_{i}\right), z_{4}=x\left(t_{j}\right)$ and let $B=\left(b_{k m}\right)$ denote their covariance matrix as in $\S 1$. Then $b_{11}=a_{i i}, b_{22}=a_{i j}, b_{33} b_{44}-b_{34}^{2}=R\left(t_{i}, t_{i}\right) R\left(t_{j}, t_{j}\right)-R\left(t_{i}, t_{j}\right)^{2}$. From the assumption on $R(s, t)$, it is clear that

$$
b_{k m}= \begin{cases}O\left(\Delta t_{i}\right), & k=1, m=3,4 ; m=1, k=3,4, \\ O\left(\Delta t_{j}\right), & k=2, m=3,4 ; m=2, k=3,4 .\end{cases}
$$

Therefore, after expanding each cofactor $B_{k m}, 1<k, m<4,(2.1)$ and (2.3) imply that

$$
\begin{gathered}
B_{11}=a_{i j} D+O\left(\Delta t_{j}^{2}\right), \quad B_{22}=a_{i i} D+O\left(\Delta t_{i}^{2}\right), \\
B_{12}=a_{i j} D+O\left(\Delta t_{i} \Delta t_{j}\right), \quad B_{k m}=O\left(\Delta t_{i} \Delta t_{j}\right), \quad k=1,2, m=3,4, \\
B_{11} B_{22}-B_{12}^{2}=\left(a_{i i} a_{j j}-a_{i j}^{2}\right) D^{2}+O\left(\Delta t_{i}^{2} \Delta t_{j}\right)+O\left(\Delta t_{i} \Delta t_{j}^{2}\right), \\
B_{12} B_{23}-B_{13} B_{22}=O\left(\Delta t_{i}^{2} \Delta t_{j}\right)=B_{12} B_{24}-B_{14} B_{22}, \\
B_{12} B_{13}-B_{11} B_{23}=O\left(\Delta t_{i} \Delta t_{j}^{2}\right)=B_{12} B_{14}-B_{11} B_{24}, \\
|B|=a_{i i} a_{j j} D+O\left(\Delta t_{i}^{2} \Delta t_{j}^{2}\right)=\left(a_{i i} a_{j j}-a_{i j}^{2}\right) D+O\left(\Delta t_{i}^{2} \Delta t_{j}^{2}\right),
\end{gathered}
$$

where $D=b_{33} b_{44}-b_{34}^{2}$. From (1.1), (2.1), (2.2), (2.4) and the fact that $f\left(t_{i}\right)>\varepsilon$, $f\left(t_{j}\right) \geqslant \varepsilon$, we see that

$$
\begin{aligned}
& m_{1}=O\left(\Delta t_{i}\right) z_{3}+O\left(\Delta t_{i}\right) z_{4}, \quad m_{2}=O\left(\Delta t_{j}\right) z_{3}+O\left(\Delta t_{j}\right) z_{4}, \\
& V_{1}=a_{i i}+O\left(\Delta t_{i}^{2}\right), \quad V_{2}=a_{j j}+O\left(\Delta t_{j}^{2}\right), \quad \Gamma=O\left(\Delta t_{i} \Delta t_{j}\right) .
\end{aligned}
$$

LemMa 2.2. Let $(i, j) \in I_{e}$. Then

$$
\begin{aligned}
E\left\{\Delta x\left(t_{i}\right)^{2} \Delta x\left(t_{j}\right)^{2} \mid x\left(t_{i}\right)=z_{3}, x\left(t_{j}\right)=z_{4}\right\} & \\
= & a_{i i} a_{j j}+O\left(\Delta t_{i}^{2} \Delta t_{j}\right)+O\left(\Delta t_{i} \Delta t_{j}^{2}\right)+P_{1}\left(z_{3}, z_{4}\right)
\end{aligned}
$$


where $P_{1}\left(z_{3}, z_{4}\right)$ is a polynomial of $z_{3}, z_{4}$ of degree 4 with coefficients of order $\Delta t_{i}^{2} \Delta t_{j}$ or $\Delta t_{i} \Delta t_{j}^{2}$.

Proof. From Property 1.2 and Property 1.3, we obtain

$$
\begin{aligned}
E\left\{\Delta x\left(t_{i}\right)^{2} \Delta x\right. & \left.\left(t_{j}\right)^{2} \mid x\left(t_{i}\right)=z_{3}, x\left(t_{j}\right)=z_{4}\right\} \\
& =\iint_{R^{2}} z_{1}^{2} z_{2}^{2} N\left(z_{1}, z_{2} \mid z_{3}, z_{4}\right) d z_{1} d z_{2} \\
& =V_{1} V_{2}+2 \Gamma^{2}+m_{1}^{2} V_{2}+m_{2}^{2} V_{1}+4 m_{1} m_{2} \Gamma+m_{1}^{2} m_{2}^{2} .
\end{aligned}
$$

Lemma 2.2 follows clearly from (2.6), (2.5) and (2.1).

By using a similar argument and the identity $E X^{2}=\operatorname{Var}(X)+(E X)^{2}$, we can show

Lemma 2.3. Let $(i, j) \in I_{\varepsilon}$. Then

$$
\begin{aligned}
& E\left\{\Delta x\left(t_{i}\right)^{2} \mid x\left(t_{i}\right)=z_{3}, x\left(t_{j}\right)=z_{4}\right\}=a_{i i}+O\left(\Delta t_{i}^{2}\right)+P_{2}\left(z_{3}, z_{4}\right), \\
& E\left\{\Delta x\left(t_{j}\right)^{2} \mid x\left(t_{i}\right)=z_{3}, x\left(t_{j}\right)=z_{4}\right\}=a_{j j}+O\left(\Delta t_{j}^{2}\right)+P_{3}\left(z_{3}, z_{4}\right),
\end{aligned}
$$

where $P_{2}, P_{3}$ are homogeneous polynomials of degree 2. All of their coefficients are of order $\Delta t_{i}^{2}$ and $\Delta t_{j}^{2}$ respectively.

Let $e_{i j}=G\left(x\left(t_{i}\right)\right) G\left(x\left(t_{j}\right)\right)\left(\Delta x\left(t_{i}\right)^{2}-a_{i i}\right)\left(\Delta x\left(t_{j}\right)^{2}-a_{j j}\right)$. Then

COROllary 2.4. Let $G$ be as in Theorem 2.1. Then

$$
\lim _{n \rightarrow \infty} \sum_{(i, j) \in I_{e}} E\left\{e_{i j}\right\}=0 .
$$

Proof. Lemma 2.2 together with Lemma 2.3 imply that, for $(i, j) \in I_{\varepsilon}$,

$$
\begin{aligned}
E\left\{e_{i j}\right\} & =E\left(E\left\{e_{i j} \mid x\left(t_{i}\right), x\left(t_{j}\right)\right\}\right) \\
& =E\left\{G\left(x\left(t_{i}\right)\right) G\left(x\left(t_{j}\right)\right)\left[O\left(\Delta t_{i}^{2} \Delta t_{j}\right)+O\left(\Delta t_{i} \Delta t_{j}^{2}\right)+P_{4}\left(x\left(t_{i}\right), x\left(t_{j}\right)\right)\right]\right\},
\end{aligned}
$$

where $P_{4}\left(z_{3}, z_{4}\right)$ is a polynomial of $z_{3}, z_{4}$ of degree 4 with all its coefficients of order $\Delta t_{i}^{2} \Delta t_{j}$ or $\Delta t_{i} \Delta t_{j}^{2}$. The assumption on $G$ implies that $E\left\{G(x(s)) G(x(t))[x(s)]^{p}[x(t)]^{q}\right\}$ is bounded for fixed integers $p, q>0$. Therefore, $E\left\{e_{i j}\right\}=O\left(\Delta t_{i}^{2} \Delta t_{j}\right)+O\left(\Delta t_{i} \Delta t_{j}^{2}\right)$. Summing up (2.7) over $(i, j) \in I_{\varepsilon}$ and letting $n \rightarrow \infty$, Corollary 2.4 follows.

LeMMA 2.5. Let $I_{1}=\left\{(i, j):\left|t_{i}-t_{j}\right|<\varepsilon\right\}$. Then

$$
\varlimsup_{n \rightarrow \infty}\left|\sum_{(i, j) \in I_{1}} E\left\{e_{i j}\right\}\right|<\text { constant } \cdot \varepsilon .
$$

Proof. By applying the Cauchy-Schwarz inequality twice, we obtain

$$
\begin{aligned}
\left|E\left\{e_{i j}\right\}\right| \leqslant & \left\{E\left[G\left(x\left(t_{i}\right)\right)^{2} G\left(x\left(t_{j}\right)\right)^{2}\right]\right\}^{1 / 2}\left\{E\left[\Delta x\left(t_{i}\right)^{2}-a_{i i}\right]^{4}\right\}^{1 / 4} \\
& \cdot\left\{E\left[\Delta x\left(t_{j}\right)^{2}-a_{j j}\right]^{4}\right\}^{1 / 4} .
\end{aligned}
$$


Let $M=\sup \left\{\left[E\left\{G(x(t))^{2} G(x(s))^{2}\right\}\right]^{1 / 2} ; 0<s, t<T\right\}$. Then $M$ is finite. Property 1.1 and (2.8) imply that

$$
\left|E\left\{e_{i j}\right\}\right| \leqslant M c_{4}^{2} a_{i i} a_{j j}=O\left(\Delta t_{i} \Delta t_{j}\right) .
$$

Therefore,

$$
\left|\sum_{(i, j) \in I_{1}} E\left\{e_{i j}\right\}\right| \leqslant \sum_{i} \sum_{\left\{j:\left|t_{j}-t_{i}\right|<\varepsilon\right\}}\left|E\left\{e_{i j}\right\}\right|<O(1) \cdot \varepsilon .
$$

Lemma 2.5 follows by letting $n \rightarrow \infty$ in the above inequality.

LEMmA 2.6. Let $I_{2}=\left\{(i, j): f\left(t_{i}\right)<\varepsilon\right.$ or $\left.f\left(t_{j}\right)<\varepsilon\right\}$. Then

$$
\overline{\lim }_{n \rightarrow \infty}\left|\sum_{(i, j) \in I_{2}} E\left\{e_{i j}\right\}\right| \leqslant \text { constant } \cdot \varepsilon .
$$

Proof. Let $(i, j) \in I_{2}$ and suppose that $f\left(t_{i}\right)<\varepsilon$. From (2.1) and the first inequality of (2.9), we see that

$$
\left|E\left\{e_{i j}\right\}\right| \leqslant M c_{4}^{2} a_{i i} a_{j j}<\varepsilon O\left(\Delta t_{i} \Delta t_{j}\right)+o\left(\Delta t_{i} \Delta t_{j}\right) .
$$

Note that (2.10) also holds if $(i, j) \in I_{2}$ and $f\left(t_{j}\right)<\varepsilon$. Hence, after summing up (2.10) over $(i, j) \in I_{2}$ and passing $n \rightarrow \infty$, Lemma 2.6 follows.

From Corollary 2.4, Lemma 2.5 and Lemma 2.6, we see that

$$
L^{2}-\lim _{n \rightarrow \infty} \sum_{k} G\left(x\left(t_{k}\right)\right) \Delta x\left(t_{k}\right)^{2}=L^{2}-\lim _{n \rightarrow \infty} \sum_{k} G\left(x\left(t_{k}\right)\right) a_{k k}
$$

if the $L^{2}$-limit in the R.H.S. of (2.11) exists. This limit exists since $E\{G(x(s)) G(x(t))\}$ is continuous in $(s, t), a_{k k}=f\left(t_{k}\right) \Delta t_{k}+o\left(\Delta t_{k}\right)$, and $f(t)$ is bounded continuous. This limit is denoted by $\int_{0}^{T} G(x(t)) f(t) d t$. This completes the proof of Theorem 2.1.

RemarK 1. Condition (R3) is equivalent to the statement that $x(s), x(t)$ are linearly independent for $s \neq t$. In the proofs of Lemmas 2.2-2.3, if $x\left(t_{i}\right), x\left(t_{j}\right)$ are linearly dependent, then we use Property 1.4 instead of Property 1.3. It would not affect the result of Lemmas 2.2-2.3. Therefore, Corollary 2.4 and, hence, Theorem 2.1 are still valid if we drop condition (R3).

REMARK 2. From the process of the proof of Theorem 2.1, we see that condition (R2) can be weakened to the statement that "For any $\varepsilon>0$, there exists $M_{\varepsilon}$ such that

$$
|R(t+d t, s+d s)-R(t+d t, s)-R(t, s+d s)+R(t, s)|<M_{e}|d t d s|
$$

uniformly for $s \neq t$ except on a set with Lebesgue measure smaller than $\varepsilon$ ". A sufficient condition for this is that $R(s, t)$ is of bounded variation.

3. Application. Let $H(y)$ be a function of $y$. We shall denote $\int_{s}^{t} H(x(r)) d x(r)$ as the $L^{2}$-limit of $\Sigma_{k} H\left(x\left(t_{k}\right)\right) \Delta x\left(t_{k}\right)$ along any sequence of partitions of $[s, t]$ whose mesh goes to 0 , when the limit exists.

Let $U(x)$ be a function having bounded continuous $U^{\prime}(x), U^{\prime \prime}(x)$ and bounded $U^{\prime \prime \prime}(x)$. Then for $0 \leqslant s<t \leqslant T$, 


$$
\begin{aligned}
U(x(t))-U(x(s))= & \sum_{k=0}^{n-1} U^{\prime}\left(x\left(t_{k}\right)\right) \Delta x\left(t_{k}\right)+\frac{1}{2} \sum_{k=0}^{n-1} U^{\prime \prime}\left(x\left(t_{k}\right)\right) \Delta x\left(t_{k}\right)^{2} \\
& +\frac{1}{6} \sum_{k=0}^{n-1} U^{\prime \prime \prime}\left(y_{k}\right) \Delta x\left(t_{k}\right)^{3}=J_{1}+J_{2}+J_{3},
\end{aligned}
$$

where $t_{k}=s+k(t-s) / n, 0 \leqslant k \leqslant n, \Delta x\left(t_{k}\right)=x\left(t_{k+1}\right)-x\left(t_{k}\right), 0<k<n-1$, and where $y_{k}$ is between $x\left(t_{k}\right)$ and $x\left(t_{k+1}\right)$ for each $k$. Using the fact that $E \Delta x\left(t_{k}\right)^{2 m}=O\left(a_{k k}^{m}\right)=O\left(\Delta t_{k}^{m}\right)$ uniformly in $k$ for each $m>1$ and the CauchySchwarz inequality, we obtain

$$
\begin{aligned}
\lim _{n \rightarrow \infty} E\left\{J_{3}^{2}\right\} & \leqslant\|U\|_{\infty}^{2} \lim _{n \rightarrow \infty} \sum_{k, j=0}^{n-1} E\left\{\left|\Delta x\left(t_{k}\right)\right|^{3}\left|\Delta x\left(t_{j}\right)\right|^{3}\right\} \\
& \leqslant\|U\|_{\infty}^{2} \lim _{n \rightarrow \infty} \sum_{k, j=0}^{n-1} O\left(\Delta t_{k}^{3 / 2}\right) O\left(\Delta t_{j}^{3 / 2}\right)=0,
\end{aligned}
$$

where $\|U\|_{\infty}$ is the supremum norm of $U$. Letting $n \rightarrow \infty$ in (3.1), Theorem 2.1 and (3.2) imply that $J_{1}$ converges in $L^{2}$. Denoting the limit by $\int_{s}^{t} U^{\prime}(x(r)) d x(r)$, we have

$$
U(x(t))-U(x(s))=\int_{s}^{t} U^{\prime}(x(r)) d x(r)+\frac{1}{2} \int_{s}^{t} U^{\prime \prime}(x(r)) f(r) d r .
$$

A slight modification of the above proof shows that (3.3) holds also for the polynomial $U(x)$. The formal differential rule is

TheOREM 3.1. Let $U(t, x), 0 \leqslant t \leqslant T, x \in R$, have bounded continuous $U_{1}^{\prime}(t, x)$, $U_{2}^{\prime}(t, x), U_{22}^{\prime \prime}(t, x)$ and bounded $U_{222}^{\prime \prime \prime}(t, x)$ or be a polynomial of $x$ with all the coefficients continuously differentiable with respect to $t$. Then, for $0<s<t<T$

$$
\begin{aligned}
U(t, x(t))= & U(s, x(s))+\int_{s}^{t} U_{2}^{\prime}(r, x(r)) d x(r) \\
& +\int_{s}^{t}\left\{U_{1}^{\prime}(r, x(r))+\frac{1}{2} U_{22}^{\prime \prime}(r, x(r)) f(r)\right\} d r .
\end{aligned}
$$

REMARK 3. The differential formula for a Gaussian process $x(t)$ given in [3, (8.1)] looks different from (3.4). This is because the definitions of $\int_{s}^{t} U(x(r)) d x(r)$ are different. Note that in $[3,(8.1)], U(t, x)$ is $\infty$-differentiable w.r.t. $x$ and in Theorem 3.1 , it is thrice differentiable w.r.t. $x$ only.

ACKNOWLEDGement. The author wishes to thank the referee for his comments.

\section{REFERENCES}

1. G. E. Baxter, A strong limit theorem for Gaussian processes, Proc. Amer. Math. Soc. 7 (1956), 522-527.

2. G. A. Brosmler, Quadratic variation of potentials and harmonic functions, Trans. Amer. Math. Soc. 149 (1970), 243-257.

3. S. T. Huang and S. Cambanis, Gaussian processes: Nonlinear analysis and stochastic calculus, Lecture Notes in Math., vol. 695, Springer-Verlag, Berlin and New York, 1974/75.

4. E. Wong and M. Zakai, The oscillation of stochastic integrals, Z. Wahrsch. Verw. Gebiete 4 (1965), 103-112.

5. A. T. Wang, Quadratic variation of functionals of Brownian motion, Ann. Probab. 5 (1977), 756-769.

6. J. Yeh, Stochastic processes and the Wiener integral, Marcel Dekker, New York, 1973.

Department of Business Mathematics, Soochow University, TaIPei, Tarwan, Republic of China 